\title{
Advances in functional limb reconstruction in the irradiated setting
}

\author{
Nicholas Marsden, Damien Grinsell \\ Department of Plastic Surgery, St Vincent's Hospital, Melbourne 3065, Australia.
}

Correspondence to: Dr. Damien Grinsell, Department of Plastic Surgery, St Vincent's Hospital, 41 Victoria Parade, Fitzroy, Melbourne, Australia. E-mail: damiengrinsell@gmail.com

How to cite this article: Marsden N, Grinsell D. Advances in functional limb reconstruction in the irradiated setting. Plast Aesthet Res 2019;6:24. http://dx.doi.org/10.20517/2347-9264.2019.14

Received: 21 Aug 2019 First Decision: 31 Oct 2019 Revised: 6 Nov 2019 Accepted: 15 Nov 2019 Published: 29 Nov 2019

Science Editor: Matthew L. Iorio Copy Editor: Jing-Wen Zhang Production Editor: Jing Yu

\begin{abstract}
The management of extremity soft tissue sarcoma is constantly evolving, and, in recent decades, limb salvage has been the main goal. More commonly, this is being achieved with a combination of neo-adjuvant radiotherapy, followed by wide excision and soft tissue reconstruction in the form of vascularised soft tissue transfer. Although limb salvage is now readily achievable, the resultant functional disabilities following excision of major musculotendinous and neurovascular structures can be life changing. In recent years, there has been a move towards functional limb reconstruction in the form of free functioning muscle transfer. This paper reviews the advances in functional limb reconstruction in the setting of preoperative radiation and reports our experience in this challenging reconstructive field.
\end{abstract}

Keywords: Sarcoma, radiation, functional limb reconstruction, free functioning muscle transfer, nerve transfer

\section{INTRODUCTION}

Soft tissue sarcomas (STS) are a rare group of mesenchymal tumours commonly affecting the extremities. Historically, extremity STS patients were commonly treated by amputation, with rates of around $40 \%-50 \%{ }^{[1]}$. However, in the 1980s, "limb-preserving surgery" became the mainstay of treatment after Rosenberg et al. ${ }^{[2]}$ demonstrated equivalent five-year survival rates with a combination of wide excision and radiotherapy compared to amputation. Limb preserving surgery in combination with radiotherapy for high-risk extremity STS has been shown to yield superior local control over excision alone ${ }^{[3-5]}$. There remains a debate

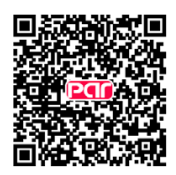


over the optimum timing of radiotherapy in the management of extremity STS. Preoperative radiotherapy is associated with better overall survival but higher wound complications compared to postoperative radiation $^{[6]}$. The most important factor in obtaining local control is wide surgical excision margins ${ }^{[7]}$, which often involves the loss of major musculotendinous and neurovascular units in the extremities. These complex defects are further complicated by the issues of radiation related wound complications, which has led to the popularisation of free and pedicled flaps to reconstruct extremity STS defects. The main benefits of importing vascularised tissue in the form of free/pedicled flaps is to fill dead space, attain wound closure, protect important neurovascular structures, and improve wound healing ${ }^{[8,9]}$. Despite these advances in reconstruction, a large proportion of patients will rely on splints and orthotics to aid activities of daily living, and the overall function remains poor.

More recently, there has been a paradigm shift in the goals of STS reconstruction, to a more innovative, functional approach, whereby the aim is to replace missing elements (e.g., skin, bone, tendon, muscle and neurovascular structures), restore functional muscle units and critical sensory pathways and provide soft tissue coverage in one procedure ${ }^{[10-13]}$. The aim of this paper is to review current innovative techniques of functional limb reconstruction in the irradiated setting, and present our experience in this challenging field.

\section{PERIPHERAL NERVE RECONSTRUCTION}

Wide excision of composite tissue in sarcoma surgery may lead to segmental loss of critical sensory and motor nerves, with devastating functional loss. There are several techniques described for reconstructing nerve gaps, whereby direct epineural repair is not an option, including autologous nerve grafts, allografts and nerve conduits. The difficulty in the post radiation setting is the poor vascularity of the wound beds, leading to generally poor results with conventional techniques.

Although the results of functional recovery after nerve repair and nerve grafts had historically been attributed to irreversible muscle atrophy and the replacement of muscle with fat over time, research has demonstrated that this is not the sole factor responsible and progressive Schwann cells denervation, nerve ischaemia, intraneural fibrosis and chronic axotomy also play significant roles ${ }^{[14]}$. The vascularised nerve graft (VNG), described by Taylor and Ham ${ }^{[15]}$, involves the transfer of a donor nerve along with its vascular pedicle. Transfer of a vascularised nerve graft avoids the initial period of nerve ischaemia and reduces central necrosis and intraneural fibrosis seen particularly in medium- to large-sized non-vascularised grafts $^{[16]}$. It is generally believed that VNGs perform better for longer gaps, larger diameter nerves and in the setting of poorly vascularised or scarred beds, however high-quality evidence is lacking. Improved nerve regeneration has been demonstrated with VNGs over standard nerve grafts in animal models in the setting of poorly vascularised beds ${ }^{[17,18]}$. One of the suggested indications for the use of vascularised nerve grafts is the poorly vascularised and scarred bed, such as in the setting of prior radiotherapy, whereby success with standard nerve grafts is generally poor; however, there is not yet firm clinical evidence for this ${ }^{[19,20]}$.

As a general rule, there is a $50 \%$ loss of axons at each nerve coaptation site. Therefore, with primary nerve repair, approximately $50 \%$ of the original axons will successfully regenerate across the repair. With nerve grafts, because of two coaptation sites, only around $25 \%$ of axons will regenerate successfully across the distant coaptation, and there may be additional axonal loss depending on the distance to the distal target, due to the effects of chronic axotomy and muscle fibrosis ${ }^{[16,21]}$. For this reason, nerve transfers, requiring a single coaptation, are favoured over nerve grafts when possible, and nerves with higher axonal input are favoured, to maximise axonal regeneration distally. Nerve transfer involves the coaptation of an expendable healthy donor nerve to a denervated or cut nerve, with the aim of maximising functional recovery with 
Table 1. Summary of the differences between nerve grafts and nerve transfers

\begin{tabular}{ll}
\hline Nerve graft & \multicolumn{1}{c}{ Nerve transfer } \\
\hline 2 Coaptations & 1 Coaptation \\
$\sim 25 \%$ of available axons to target & $\sim 50 \%$ of available axons to target \\
Longer distance to target & Shorter distance to target \\
Longer time to reinnervation & Shorter time to reinnervation \\
Higher chance of motor end plate degeneration & Allows for delayed reconstruction \\
Less specific & Highly specific \\
Donor site morbidity & Micro-neurolysis can preserve donor function \\
\hline
\end{tabular}

faster reinnervation to distal targets ${ }^{[22]}$. Most commonly, this nerve transfer is used to restore motor function, but it can be used to restore critical sensory function. The advantages of nerve transfer over nerve grafts are well documented in the literature and are summarised in Table 1.

The combination of peripheral nerve reconstruction, such as nerve graft or transfer, along with importing healthy vascularised tissue coverage in the form of free or pedicled tissue transfer is a useful technique to optimise the local environment for nerve regeneration. Nerve grafts and transfers play a significant role in extremity reconstruction and, depending on the defect characteristics (e.g., resection of major nerves and muscular units), can be employed in combination with newer microsurgical techniques that have developed in recent decades.

\section{FUNCTIONING MUSCLE TRANSFER}

Functioning muscle transfer (FMT) involves the transfer of a healthy donor muscle and its neurovascular pedicle to a new location to assume a new function. Free functioning muscle transfer (FFMT) involves restoring the circulation of the transferred muscle with microsurgical anastomosis to vessels at the recipient site along with coaptation of the motor nerve. Pedicled innervated flaps maintain their vascular supply but involve reorientation of the muscle and reinnervation from nerve transfer at the recipient site with a view to altering the function of that muscle. Within several months, the transferred muscle becomes reinnervated by the donor nerve, eventually begins to contract and ultimately gains independent function ${ }^{[23]}$. FMT has traditionally been limited to muscles with a single nerve for transfer (e.g., gracillis), because of the view that segmental nerve supply would be an obstacle to reinnervation. However, we have previously published a series of 11 functional quadriceps reconstructions using innervated rectus abdominis flaps, whereby 2-3 segmental nerves have been coaptated to cut femoral nerve branches at the recipient site. The overall functional results were excellent with over 50\% achieving M5 power [as per the Medical Research Council (MRC) grading system] with a mean follow-up of 12 months and with minimal donor site complications ${ }^{[24]}$. The rectus abdominis has the added versatility of being used as a pedicled innervated flap for quadriceps reconstruction, and its segmental innervation does not preclude its use as a functional muscle transfer ${ }^{[25]}$.

The most reported FFMTs in the literature for STS extremity reconstruction are the gracillis $^{[1,12,26]}$, latissimus dorsi $(\mathrm{LD})^{[11,25,26]}$ and rectus abdominis muscle [vertical (VRAM) or transverse (TRAM) ${ }^{[24]}$, with the other less commonly transferred muscles being the innervated medial gastrocnemius muscle ${ }^{[27]}$ and rectus femoris [Figures 1-4]. FFMT can be performed in combination with nerve grafts and/or nerve transfer, especially when the resection involves loss of major nerves and muscle units. Nerve grafts, either standard or vascularised, are generally used with the aim of restoring critical sensory function, whereas nerve transfer is primarily used to restore motor function.

\section{LONG DONOR NERVE HARVEST WITH FFMT}

In general, following sarcoma resection, there are usually native nerves available for coaptation to the transferred muscle flap, which is advantageous as the length of nerve available with most flaps is short. 

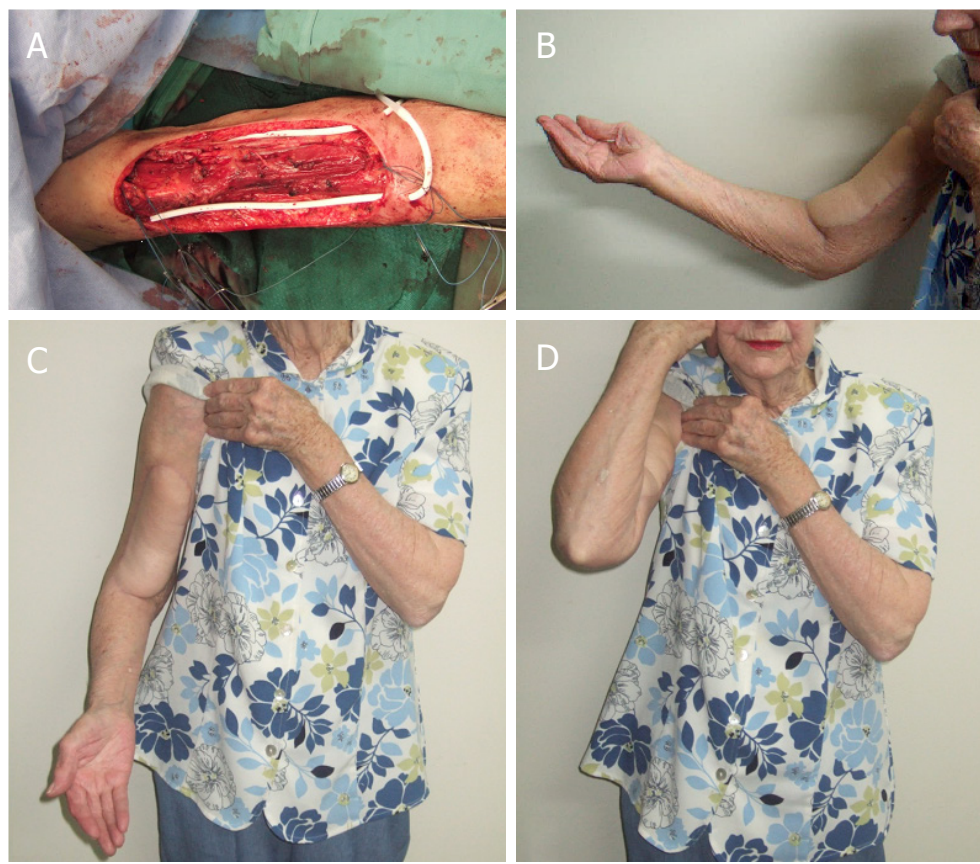

Figure 1. Clinical images of functional upper limb reconstruction with a combination of free functioning muscle transfer and nerve transfer in an 83-year-old patient. A: right arm defect following sarcoma excision including $100 \%$ of biceps and over $50 \%$ of brachialis, which was denervated. This was reconstructed with a free innervated gracillis myocutaneous flap with the nerve coaptated to the cut end of the musculocutaneous nerve, along with a flexor carpi ulnaris branch to brachialis branch nerve transfer; B: results after 12 months, demonstrating very good cosmesis and contour; C, D: active elbow flexion from $110^{\circ}$ to $40^{\circ}$ with $M 4$ power (see Video 1)
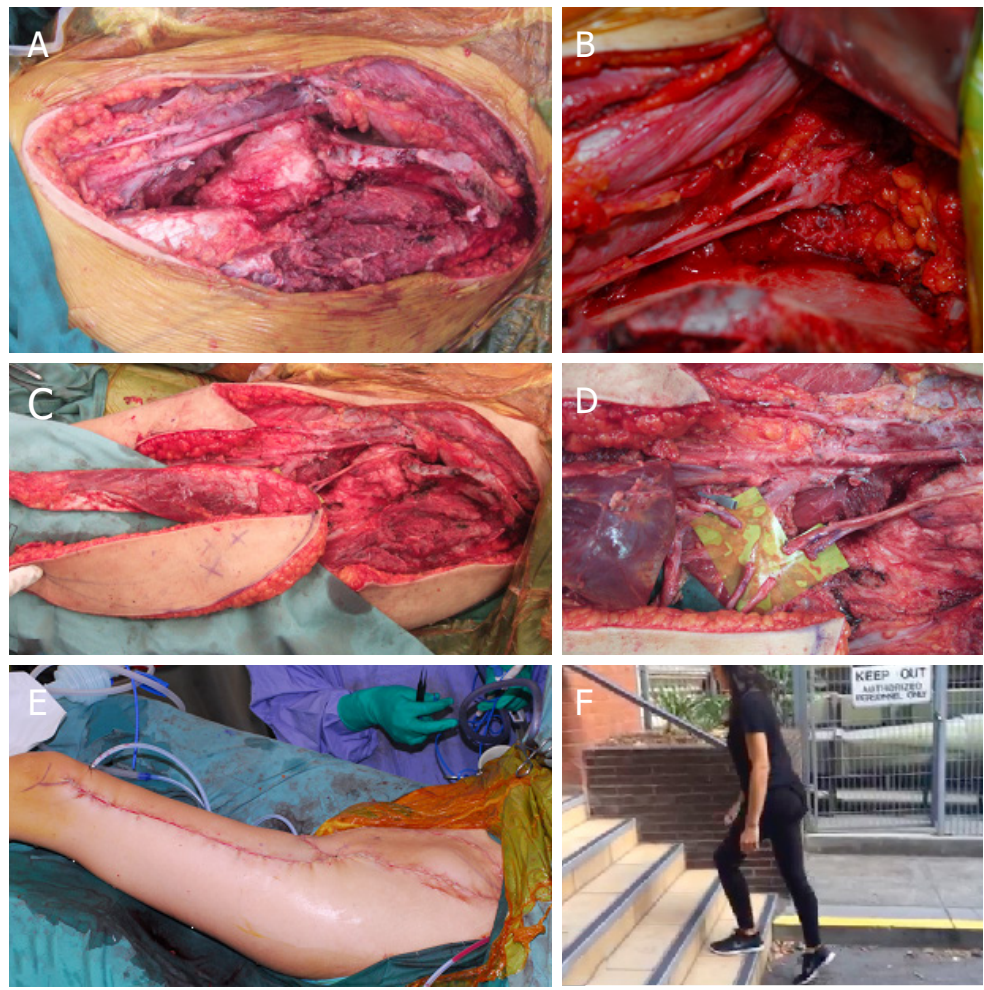

Figure 2. Example of lower limb functional reconstruction with functional muscle transfer, nerve grafts and nerve transfers in a 30-yearold female. A: large defect to left groin following excision of sarcoma, which included femoral nerve and iliopsoas and sartorius muscle resection, also demonstrating exposed femoral artery and vein; B: nerve grafts performed using cutaneous femoral nerve branches, from proximal stump to quadriceps branches; C: pedicled, innervated rectus femoris myocutaneous flap raised prior to inset to reconstruct hip flexors; D: adductor longus nerve transfer to vastus medialis oblique branch; E: final result after inset and closure; F: patient had full return of hip flexors and quadriceps function, and was able to run, climb and descend stairs at 18 months (see Videos 2 and 3) 

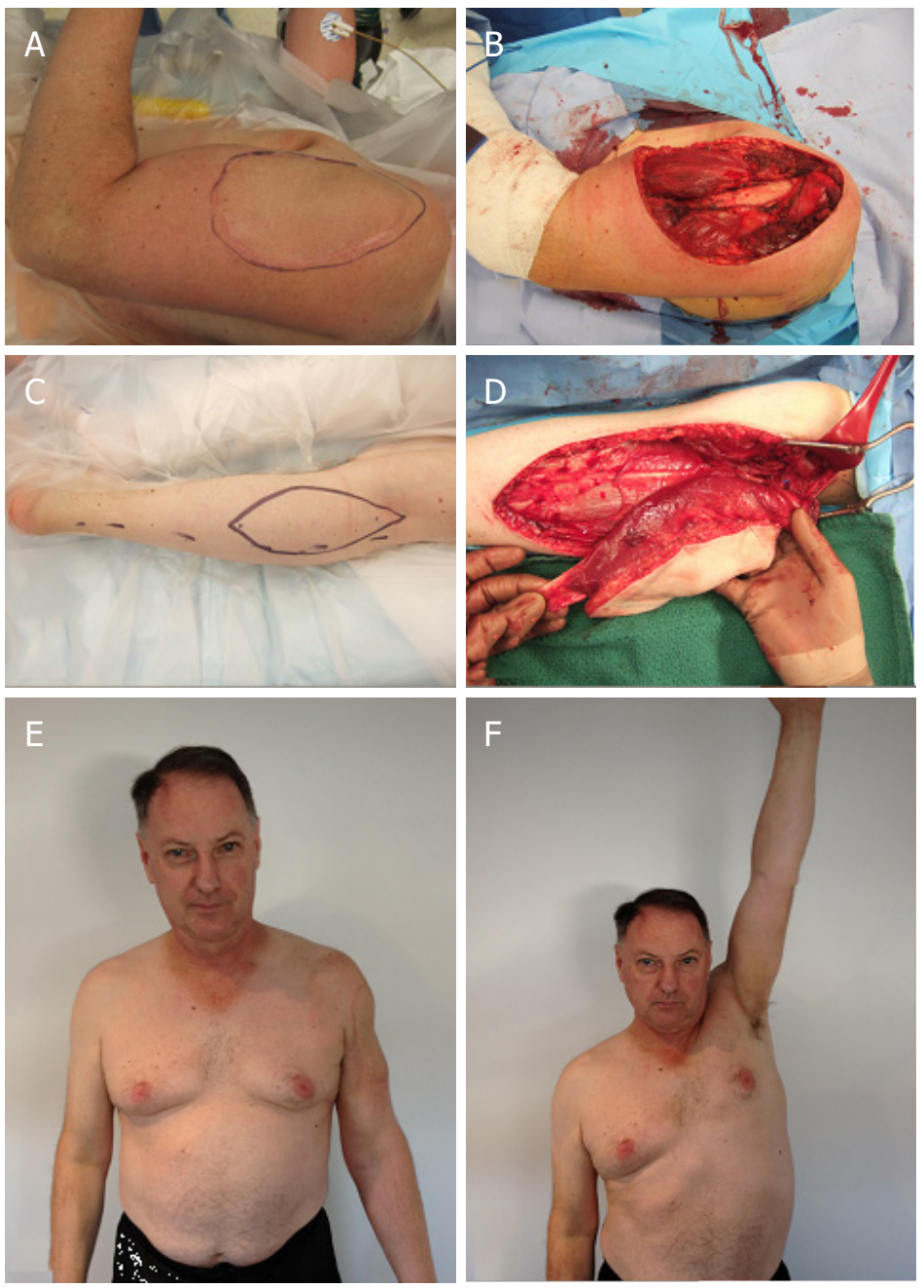

Figure 3. Clinical images of an example of upper limb functional reconstruction with an innervated medial gastrocnemius myocutaneous flap, following left deltoid excision for recurrent sarcoma (previous partial deltoid resection and ALT flap). A: planned excision of previous ALT flap and remaining deltoid; B: defect following total deltoid resection, with exposed humerus; C: right medial gastrocnemius myocutaneous flap planning; D: flap islanded with its neurovascular bundle dissected prior to division; E: result at one year showing excellent flap contour; F: M5 power of shoulder abduction and flexion, equal to the contralateral side (see Video 4). ALT: antero-lateral thigh

However, there may be circumstances following more extensive resections where longer length is required to perform reinnervation. The senior author has successfully performed FFMT with long nerve harvest in both rectus abdominis and gracillis transfer. Free or pedicled rectus abdominis myocutaneous flaps can be raised with long dissection of over $10 \mathrm{~cm}$ of intercostal nerves [Figure 5].

We have had excellent results with pedicled, innervated rectus abdominis flaps in quadriceps reconstruction, with evidence of motor function in the donor muscle within three months and MRC 5/5 return of power at seven months ${ }^{[24]}$. The most commonly used free functioning flap for both oncologic and trauma reconstruction is the gracillis flap. One of the limitations to its use is the relatively short neurovascular pedicle, usually allowing around $6-8 \mathrm{~cm}$ of length to be harvested. When longer lengths of donor nerve are required, long nerve gracillis flaps can be harvested through the obturator foramen, via a combined intra-abdominal approach, allowing up to $30 \mathrm{~cm}$ of donor nerve to be harvested with the muscle. Time to reinnervation is increased due to the longer distances for regeneration, but full function can be achieved successfully with these techniques. 

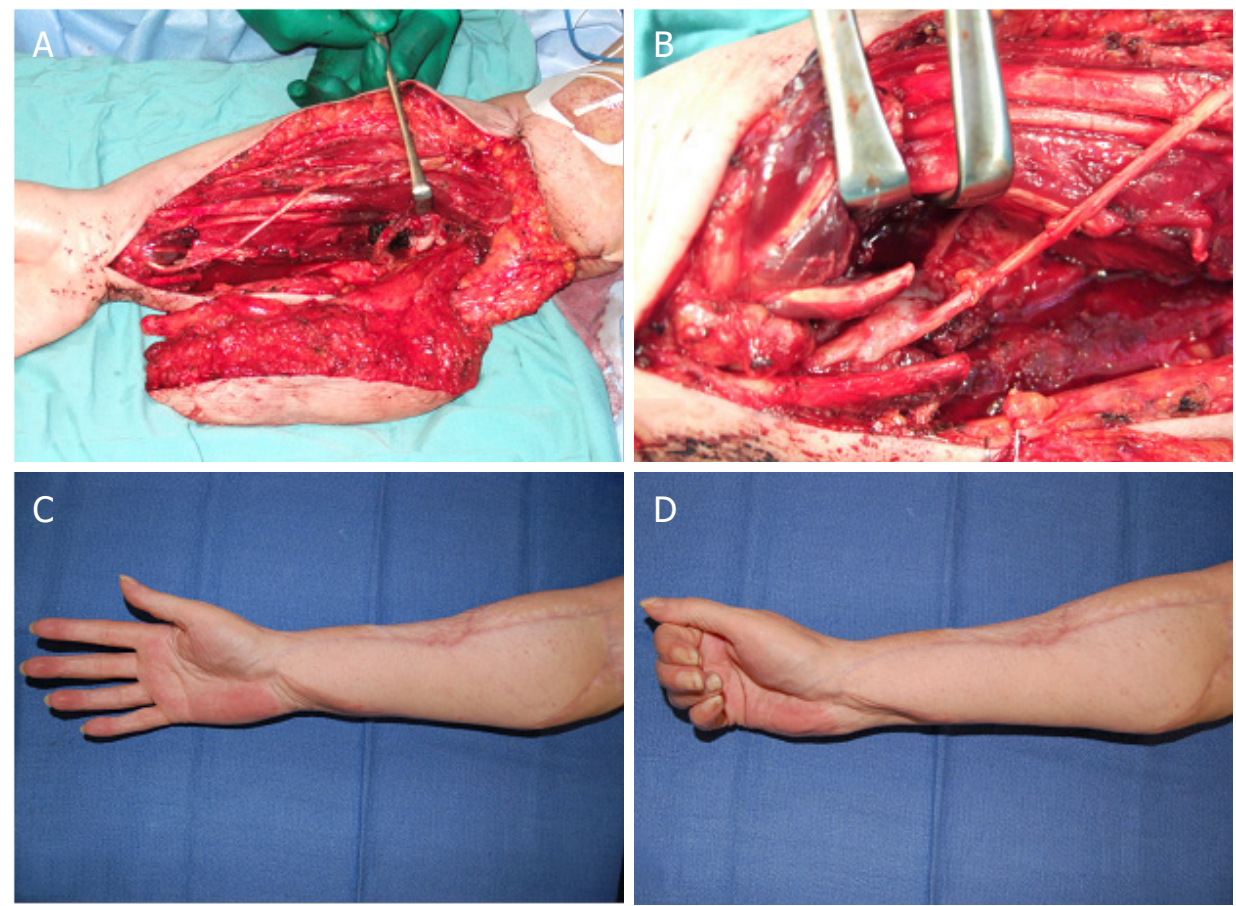

Figure 4. Functional upper limb reconstruction with a combination of free functioning muscle transfer and nerve transfer for a right forearm defect in a 68-year-old female, which involved resection of $15 \mathrm{~cm}$ of ulnar nerve and common flexor mass. A: free functioning gracillis myocutaneous flap in position with the proximal muscle inset into proximal flexor digitorum profundus stump; B: close up image demonstrating the anterior interosseous nerve to ulnar motor and lateral cutaneous nerve of the forearm to ulnar sensory nerve transfers; C: result at one year showing excellent flap contour, no claw-hand deformity; D: good flexion of digits demonstrating reinnervation of the gracillis muscle

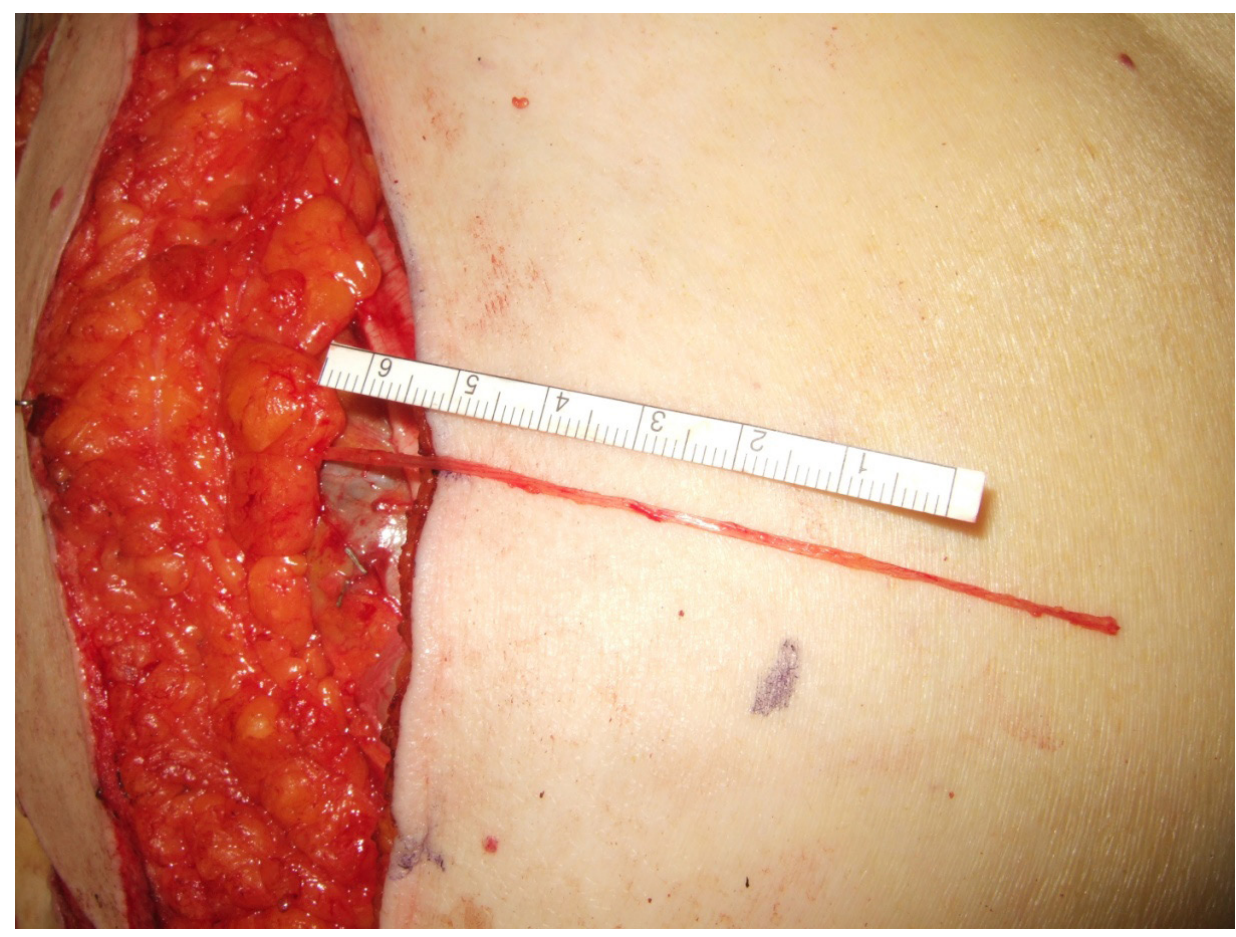

Figure 5. Innervated rectus abdominis myocutaneous flap harvest with good length of intercostal nerve available through a standard approach 


\section{OUR EXPERIENCE}

The senior author has been part of the local sarcoma multi-disciplinary team (MDT) for over 10 years, has been instrumental in advocating functional reconstruction for extremity STS in recent years and has pioneered numerous reconstructive techniques in this field.

Preoperative radiotherapy is the preferred method in our institution for the management of all extremity STS, because the sarcoma service feels the oncological outcomes are at least equivalent, and, functionally, these patients do better overall ${ }^{[25]}$. Preoperative radiotherapy is associated with better overall survival but higher wound complications compared to postoperative radiation, which we feel is overcome by the combination of wide resection and flap coverage with healthy vascularised tissue ${ }^{[6]}$. Preoperative radiotherapy allows the ability to give lower radiation doses due to improved limb perfusion and oxygenation $^{[4,28]}$, smaller radiotherapy targets ${ }^{[29]}$ and therefore decreased late toxicity ${ }^{[6]}$ compared to postoperative methods. Single stage orthoplastic surgery is performed six weeks following completion of radiotherapy, with the oncologic surgeons performing wide excision and immediate reconstruction performed by the plastic surgeons. Our unit's protocol and experience with preoperative radiotherapy have previously been reported in the literature ${ }^{[30]}$ and the results are in keeping with the current literature, which demonstrates a significant increase in complications if flaps were performed beyond this six-week period ${ }^{[31]}$.

Over the past 10 years, the senior author has developed a systematic and regimented approach to STS extremity functional reconstruction with the aim of minimising errors and maximising ergonomics and improving functional outcomes. The general approach is as follows: after tumour excision by the resecting team, haemostasis is thoroughly performed and local anatomy is examined for recipient vessels and nerves. The defect is templated, taking into account the innervated muscle requirement, alongside the need for skin and dead space filling. The flap is not detached until the recipient vessels and nerves are ready for microsurgical anastomosis in order to minimise ischaemia time. The dichotomy of neurovascular pedicle length versus vessel calibre is addressed, prior to pedicle detachment. The flap is transferred to the defect and stretched to its original length in order to allow for the final inset and the lengths of the vessels and nerves matched to the recipients. The flap is then secured proximally to prevent avulsion. The nearest motor nerve, which has been tagged during the resection, is utilised for the neurorrhaphy. The recipient nerves are stimulated intraoperatively before division with a handheld nerve stimulator in order to confirm the presence of motor axons. Anastomosis is then performed on the veins, artery and finally the nerve. The limb is then positioned appropriately depending on the compartment being reconstructed, e.g., knee extended or flexed for quadriceps and hamstring, respectively, or hip extended for gluteal reconstruction, before, finally, the distal end of the flap is tensioned and secured distally taking care to avoid tension on the anastomosis. The flap is inset in layers over suction drains and covered with a waterproof dressing. Postoperatively, the involved limb is immobilised in a fixed articulated splint for six weeks. For lower limb reconstructions, the patient is confined to bed rest for six days and nursed appropriately depending of position of the flap, before being mobilised on crutches. The uninvolved joints are allowed to move freely postoperatively, encouraging locomotion to prevent stiffness, DVT and other postoperative complications. After this six-week period, patients are allowed to start active and passive range of motion under guidance from specialist physiotherapists. Strengthening exercises are commenced after 3-6 months for a minimum period of 12 months.

Between 2009 and 2019, the senior author has performed 68 functional reconstructions, for extremity STS resections following neoadjuvant radiotherapy, including 53 free (of which two were vascularised sural nerve grafts) and 11 pedicled flaps [Table 2]. There were two patients who underwent non-vascularised nerve grafts and two who had nerve transfers alone. There were seven patients who underwent nonvascularised nerve grafts in combination with the flap coverage and four who had nerve transfers as well as flap coverage. Nerve grafts and transfers are generally used in combination with flap coverage when major 
Table 2. Summary of the various functional reconstructions performed for extremity STS

\begin{tabular}{llc}
\hline Flap & \multicolumn{1}{c}{ Defects } & Number \\
\hline LD & Gluteal, hamstrings, quadriceps, gastroc/soleus & 21 \\
Gracillis & Hamstring, quadriceps, adductors, tibialis ant, biceps/brachialis, triceps & 14 \\
VRAM-Free & Quadriceps, adductors & 10 \\
-Pedicled & & 10 \\
TRAM & Quadriceps, gluteals & 4 \\
Medial gastrocnemius & Deltoid, biceps/brachialis & 2 \\
Vascularised sural nerve & Common peroneal nerve, posterior cord & 2 \\
Rectus femoris (pedicled) & Groin (including femoral nerve and hip flexors) & 1 \\
\hline
\end{tabular}

STS: soft tissue sarcomas; LD: latissimus dorsi; VRAM: vertical rectus abdominis muscle; TRAM: transverse rectus abdominis muscle

nerves are sacrificed as part of the oncologic resection (as shown in Figures 1,2 and 4). In this series, the most commonly resected nerves were the sciatic and femoral nerve, with one case of common peroneal nerve, one ulnar nerve and one posterior cord resection for malignant peripheral nerve sheath tumour.

The mean age of patient was 63 (in the range of 35-87 years). Ninety percent of the reconstructions were of the lower limb, most commonly the quadriceps, followed by the hamstring and gluteal compartments, and $10 \%$ were of the upper limb. There were three complete flap losses (4.4\%) and one partial flap loss (1.5\%) in the series. Reinnervation was seen in the transferred muscle as early as three months postoperatively, with a mean time of 12 months (follow up by the senior surgeon occurred at six weeks, and then 3, 6, 12 and 24 months, thus exact time points of reinnervation are estimates within these timeframes). The mean MRC grade achieved was $4 / 5$, with over 50\% $(n=32)$ achieving MRC $5 / 5$ at latest follow up. Seven of the cases are too early in their follow-up to ascertain the level of functional recovery at the time of writing. With regards to nerve reconstruction, there are two patients with adequate follow-up who have recovered some protective sensation distally (one vascularised sural nerve graft and flexor carpi ulnaris (FCU) nerve branch transfer to triceps for a right posterior cord sarcoma and one sural to tibial nerve transfer following tibial nerve resection). The first patient has had an excellent result with M5/5 power of deltoid and triceps, and wrist and finger extension at 18 months (see Videos 5 and 6). A third patient who underwent vascularised sural nerve graft for common peroneal nerve (CPN) resection showed signs of sensory recovery five months postoperatively, and we await longer-term follow-up to assess final outcome.

The senior author's philosophy on functional limb reconstruction is: age is not a barrier to reconstruction (see Figure 1); the status of the joints proximal and distal to the defect are vital; aim to perform a single nerve coaptation either via nerve transfer or as part of an innervated free flap; and high axonal input is key to proximal nerve reconstruction.

\section{DISCUSSION}

Microsurgical reconstruction after soft tissue sarcoma excision has expanded the indications for limb salvage by allowing wider excision margins with the ability to adequately reconstruct the defect. However, limb salvage surgery with oncological resection of extremity STS often leads to a significant detrimental effect on mobility and the ability to perform activities of daily living, which has been shown to reduce patient's quality of life ${ }^{[32]}$. Functional reconstruction of extremities following STS excision with FFMT can provide the dual functions of active muscle contraction and soft tissue coverage in one operation.

The concept of limb salvage surgery has evolved from just anatomical preservation of the limb to preservation with restoration of function and aesthetics. Despite this paradigm shift in recent years, functional reconstruction following extremity sarcoma resection is still relatively uncommon. A recently published review reported just 134 cases of functional sarcoma reconstruction of the limbs in the literature, 
of which only 55 were FFMT and 17 were nerve reconstructions, with the remainder comprising tendon transfers ${ }^{[23]}$. Nelson et al.$^{[33]}$ investigated the difference between functional reconstruction of the extremities following STS resection and soft tissue coverage alone. The study demonstrated that, although there was an increased cost and slightly extended surgical time associated with functional reconstruction, the postoperative functional outcome was better, and they concluded that this justified its use.

Although the effect of neo-adjuvant therapy on functional reconstruction has not been investigated, numerous studies have demonstrated that preoperative radiotherapy does not increase complications when flaps are used for reconstruction ${ }^{[34-36]}$. In addition, there appears to be no difference in outcomes and complication rates with muscle flaps compared to fasciocutaneous flaps when used in the post radiotherapy setting $^{[31]}$.

One area which continues to be a topic for debate is the reconstruction of major nerve defects, particularly of the sciatic nerve. The senior author has utilised different techniques for reconstructing major nerve gaps, including cable nerve grafts and vascularised sural nerve grafts. Our results are in keeping with those in the literature, which show mixed sensory outcomes. Tokumoto et al. ${ }^{[37]}$ reported three cases of vascularised sural nerve grafts for sciatic nerve reconstruction, whereby they aimed to selectively reconstructed sensation to the plantar surface of the foot. They demonstrated some sensory recovery to the sole in two patients; however, limited protective sensation was achieved. They stated poor results in the setting of postoperative radiation therapy, although this was only the case in one patient ${ }^{[37]}$. Melendez et al. ${ }^{[38]}$ reported five sciatic nerve reconstructions with cable sural nerve grafts. They demonstrated the return of partial distal sensory recovery in three patients and some protective sensation in the other two, with a mean follow up of one year ${ }^{[38]}$. From our experience and that of the limited reports in the literature, although the chances of marked sensory recovery are slight, the amount of reinnervation is such that attempts at nerve reconstruction are justified.

In the opinion of the senior author, the difference between raising an innervated free flap as opposed to a non-innervated free flap is small, especially in the case of the latissimus dorsi and gracillis flap, where the nerves lie in close proximity to the vascular pedicle. Although dissecting out recipient nerves and appropriately securing and tensioning the musculotendinous components are critical in achieving a good outcome, this is not hugely time-consuming and can be learned quickly.

As Martin et al. ${ }^{[23]}$ concluded in their review paper, there is a significant lack of high-level evidence regarding the use of functional reconstruction in extremity sarcoma. We describe here the senior author's experience in this challenging and innovative field, and demonstrate how excellent functional outcomes can be achieved with a systematic and logical approach. However, large well-designed studies are required to clarify the differences in functional and non-functional reconstruction in terms of cost, donor morbidity and functional outcomes to cement its role in sarcoma surgery.

\section{CONCLUSIONS}

Limb sparing surgery following neo-adjuvant radiation has become the preferred treatment for extremity STS. However, adequate tumour resection can compromise critical limb function. Functional reconstruction in extremity sarcoma is a relatively new concept, with limited experience published in the literature. The use of advanced microsurgical techniques such as nerve transfer and FFMT provides the reconstructive surgeon with a way of not only salvaging limbs, but restoring function following loss of critical motor and sensory structures in upper and lower extremity sarcoma resection. We feel that the functional benefits outweigh the slightly increased cost and operative time of soft tissue only reconstruction and should be considered in patients undergoing extremity sarcoma resection following radiotherapy. 


\section{DECLARATIONS}

\section{Authors' contributions}

Made substantial contributions to conception and design of the study, performed case note review and contributed to the final manuscript: Marsden N, Grinsell D

\section{Availability of data and materials}

Not applicable.

\section{Financial support and sponsorship}

None.

\section{Conflicts of interest}

All authors declared that there are no conflicts of interest.

\section{Ethical approval and consent to participate}

Not applicable.

\section{Consent for publication}

Written, informed consent was obtained for all images and videos taken, for use in medical records, teaching and publications.

\section{Copyright}

(C) The Author(s) 2019.

\section{REFERENCES}

1 Abbas JS, Holyoke ED, Moore R, Karakousis CP. The surgical treatment and outcome of soft-tissue sarcoma. Arch Surg 1981;116:765-9.

2 Rosenberg SA, Tepper J, Glatstein E, Costa J, Baker A, et al. The treatment of soft-tissue sarcomas of the extremities: prospective randomized evaluations of (1) limb-sparing surgery plus radiation therapy compared with amputation and (2) the role of adjuvant chemotherapy. Ann Surg 1982;196:305-15.

3 Lindberg RD, Martin RG, Romsdahl MM, Barkley HT Jr. Conservative surgery and postoperative radiotherapy in 300 adults with softtissue sarcomas. Cancer 1981;47:2391-7.

4 Suit HD, Mankin HJ, Wood WC, Proppe KH. Preoperative, intraoperative and postoperative radiation in the treatment of primary soft tissue sarcomas. Cancer 1985;55:2659-67.

5 Yang JC, Chang AE, Baker AR, Sindelar WF, Danforth DN, et al. Randomized prospective study of the benefit of adjuvant radiation therapy in the treatment of soft tissue sarcomas of the extremity. J Clin Oncol 1998;16:197-203.

6 O'Sullivan B, Davies AM, Turcotte R, Bell R, Catton C, et al. Preoperative versus postoperative radiotherapy in soft-tissue sarcoma of the limbs: a randomized trial. Lancet 2002;359:2235-41.

7 Bell RS, O’Sullivan B, Liu FF. The surgical margin in soft-tissue sarcoma. J Bone Joint Surg Am 1989;71:370-5.

8 Popov P, Tukiainen E, Asko-Seljaavaara S, Huuhtanen R, Virolainen M, et al. Soft tissue sarcomas of the lower extremity: surgical treatment and outcome. Eur J Surg Oncol 2000;26:679-85.

9 Carlson GW. The evolution of extremity reconstruction for soft tissue sarcoma. Ann Surg Oncol 2006;13:610-1.

10 Doi K, Kuwata N, Kawakami F, Hattori Y, Otsuka K, et al. Limb-sparing surgery with reinnervated free-muscle transfer following radical excision of soft-tissue sarcoma in the extremity. Plast Reconstr Surg 1999;104:1679-87.

11 Muramatsu K, Ihara K, Doi K, Yoshida K, Iwanaga R, et al. Functional neuro-vascularised muscle transfer for oncological reconstruction of extremity sarcoma. Surg Oncol 2012;21:263-8.

12 Grinsell D, Di Bella C, Choong PF. Functional reconstruction of sarcoma defects utilizing innervated free flaps. Sarcoma 2012;2012:315190.

13 Stranix JT, Lee ZH, Lam G, Mirrer J, Rapp T, et al. Limb-sparing sarcoma reconstruction with functional composite thigh flaps. Microsurgery 2018;38:466-72.

14 Fu SY, Gordon T. Contributing factors to poor functional recovery after delayed nerve repair: prolonged denervation. J Neurosci 1995;15:3886-95.

15 Taylor GI, Ham FJ. The free vascularized nerve graft. A further experimental and clinical application of microvascular techniques. Plast Reconstr Surg 1976;57:413-25. 
16 Grinsell D, Keating CP. Peripheral nerve reconstruction after injury: a review of clinical and experimental therapies. Biomed Res Int 2014;2014:698256.

17 Koshima I, Harii K. Experimental study of vascularized nerve grafts: multifactoral analyses of axonal regeneration of nerves transplanted into an acute burn wound. J Hand Surg Am 1985;10:64-72.

18 Kanaya F, Firrel J, Tsai TM, Breidenbach WC. Functional results of vascularized versus nonvascularized nerve grafting. Plast Reconstr Surg 1992;5:924-30.

19 D'Arpa S, Claes KEY, Stillaert F, Coleblunders B, Monstrey S, et al. Vascularized nerve "grafts": just a graft or a worthwhile procedure? Plast Aesthet Res 2015;2:183-94.

20 Terzis JK, Kostopoulos VK. Vascularized nerve graft and vascularized fascia for upper extremity nerve reconstruction. Hand 2010;5:19-30.

21 Millesi H. Progress in peripheral nerve reconstruction. World J Surg 1990;14:733-47.

22 Lee SK, Wolfe SW. Nerve transfers for the upper extremity: new horizons in nerve reconstruction. J Am Acad Orthop Surg 2012;20:506-17.

23 Martin E, Dullaart MJ, van de Sande MAJ, van Houdt WJ, Schellekens PPA, et al. Resuscitating extremities after soft tissue sarcoma resections: are functional reconstructions an overlooked option in limb salvage? A systematic review. Eur J Surg Oncol 2019;45:1762-9.

24 Grinsell D, Lonie S, Wilson KC, Choong PF. The innervated rectus abdoninis flap for quadriceps reconstruction. J Plast Reconstr Aesthet Surg 2019;72:941-5.

25 Grinsell DG, Ahmad Z. The free innervated latissimus dorsi flap for functional reconstruction following soft tissue sarcoma resection of the posterior compartment of the thigh. Eur J Plast Surg 2019;42:371-8.

26 Doi K, Kuwata N, Kawakami F, Hattori Y, Otsuka K, et al. Limb-sparing surgery with reinnervated free-muscle transfer following radical excision of soft-tissue sarcoma in the extremity. Plast Reconstr Surg 1999;104:1679-87.

27 Grinsell D, Yue BTY. The functional free innervated medial gastrocnemius flap. J Reconstr Microsurg 2014;30:451-6.

28 Enneking WF, Spanier SS, Goodman MA. A system for the surgical staging of musculoskeletal sarcoma. Clin Orthop Relat Res 1980;106-20.

29 Nielson OS, Cummings B, O'Sullivan B, Catton C, Bell RS, et al. Preoperative and postoperative irradiationof soft-tissue sarcomas: effect on radiation field size. Int J Radiat Oncol Biol Phys 1991;21:1595-9.

30 Hui A, Ngan S, Wong K, Powell G, Choong P. Preoperative radiotherapy for soft tissue sarcoma: the Peter Macallum Cancer Centre experience. Eur J Surg Oncol 2006;32:1159-64.

31 Kadle R, Motosko CC, Zakhem GA, Stranix JT, Rapp T, et al. Flap reconstruction of sarcoma defects in the setting of neoadjuvant and adjuvant radiation. J Reconstr Microsurg 2019;35:287-93.

32 Gundle KR, Cizik AM, Jones RL, Davidson DJ. Quality of life measures in soft tissue sarcoma. Expert Rev Anticancer Ther 2015;15:95100.

33 Nelson AA, Frassica FJ, Gordon TA, Deune EG. Cost analysis of functional restoration surgery for soft-tissue sarcoma. Plast Reconstr Surg 2006;117:277-83.

34 Chao AH, Chang DW, Shuaib SW, Hanasono MM. The effect of neoadjuvant versus adjuvant irradiation on microvascular free flap reconstruction in sarcoma patients. Plast Reconstr Surg 2012;129:675-82.

35 Barwick WJ, Goldberg JA, Scully SP, Harrelson JM. Vascularised tissue transfer for closure of irradiated wounds after soft tissue sarcoma resection. Ann Surg 1992;216:591-5.

36 Townley WA, Mah E, O’Neil AC, Wunder JS, Ferguson PC, et al. Reconstruction of sarcoma defects following pre-operative radiation: free tissue transfer is safe and reliable. J Plast Reconstr Aesthet Surg 2013;66:1575-9.

37 Tokumoto H, Akita S, Kubota Y, Kuriyama M, Mitsukowa N. Use of vscularised sural nerve grafts for sciatic nerve reconstruction after malignant bone and soft tissue tumour resection in the lower legs. Ann Plast Surg 2018;80:379-83.

38 Melendez M, Brandt K, Evans GRD. Sciatic nerve reconstruction: limb preservation after sarcoma resection. Ann Plast Surg 2001;46:375-81. 